\title{
Interleukin-35 Expression in Non-Small Cell Lung Cancer is Associated with Tumor Progression
}

Mingfei Sun Xianjie Zheng Qingjiang Meng Yanjun Dong

Guoyu Zhang Dexin Rao Xiaokang An Zhongxin Yang

Lihong Pan Shuanglin Zhang

Department of Cardiovascular Thoracic Surgery, The First Affiliated Hospital of Henan University,

Kaifeng City, China 